\title{
Exponential Ergodicity of the Jump-Diffusion CIR Process
}

\author{
Peng Jin, Barbara Rüdiger and Chiraz Trabelsi
}

\begin{abstract}
In this paper we study the jump-diffusion CIR process (shorted as JCIR), which is an extension of the classical CIR model. The jumps of the JCIR are introduced with the help of a pure-jump Lévy process $\left(J_{t}, t \geq 0\right)$. Under some suitable conditions on the Lévy measure of $\left(J_{t}, t \geq 0\right)$, we derive a lower bound for the transition densities of the JCIR process. We also find some sufficient conditions under which the function $V(x)=x, x \geq 0$, is a Forster-Lyapunov function for the JCIR process. This allows us to prove that the JCIR process is exponentially ergodic.
\end{abstract}

Keywords CIR model with jumps - Exponential ergodicity · Forster-Lyapunov functions $\cdot$ Stochastic differential equations

MSC: $60 \mathrm{H} 10 \cdot 60 \mathrm{~J} 60$

\section{Introduction}

The Cox-Ingersoll-Ross model (or CIR model) was introduced in [1] by Cox et al. in order to describe the random evolution of interest rates. The CIR model captures many features of the real world interest rates. In particular, the interest rate in the CIR model is non-negative and mean-reverting. Because of its vast applications in

P. Jin $(\bowtie) \cdot$ B. Rüdiger

Fachbereich C, Bergische Universität Wuppertal, Gaußstraße 20,

42119 Wuppertal, Germany

e-mail: jin@uni-wuppertal.de

B. Rüdiger

e-mail: ruediger@uni-wuppertal.de

C. Trabelsi

Department of Mathematics, Faculty of Sciences of Tunis, University of Tunis El Manar,

Campus Universitaire Farhat Hached, 2092 Tunis, Tunisia

e-mail: trabelsichiraz@ hotmail.fr 
mathematical finance, some extensions of the CIR model have been introduced and studied, see e.g. [2, 5, 15].

In this paper we study an extension of the CIR model including jumps, the socalled jump-diffusion CIR process (shorted as JCIR). The JCIR process is defined as the unique strong solution $X:=\left(X_{t}, t \geq 0\right)$ to the following stochastic differential equation

$$
d X_{t}=a\left(\theta-X_{t}\right) d t+\sigma \sqrt{X_{t}} d W_{t}+d J_{t}, \quad X_{0} \geq 0,
$$

where $a, \sigma>0, \theta \geq 0$ are constants, $\left(W_{t}, t \geq 0\right)$ is a 1-dimensional Brownian motion and $\left(J_{t}, t \geq 0\right)$ is a pure-jump Lévy process with its Lévy measure $v$ concentrated on $(0, \infty)$ and satisfying

$$
\int_{(0, \infty)}(\xi \wedge 1) v(d \xi)<\infty,
$$

independent of the Brownian motion $\left(W_{t}, t \geq 0\right)$. The initial value $X_{0}$ is assumed to be independent of $\left(W_{t}, t \geq 0\right)$ and $\left(J_{t}, t \geq 0\right)$. We assume that all the above processes are defined on some filtered probability space $\left(\Omega, \mathscr{F},(\mathscr{F})_{t \geq 0}, P\right)$. We remark that the existence and uniqueness of strong solutions to (1) are guaranteed by [7, Theorem 5.1].

The term $a\left(\theta-X_{t}\right)$ in (1) defines a mean reverting drift pulling the process towards its long-term value $\theta$ with a speed of adjustment equal to $a$. Since the diffusion coefficient in the SDE (1) is degenerate at 0 and only positive jumps are allowed, the JCIR process $\left(X_{t}, t \geq 0\right)$ stays non-negative if $X_{0} \geq 0$. This fact can be shown rigorously with the help of comparison theorems for SDEs, for more details we refer the readers to [7].

The JCIR defined in (1) includes the basic affine jump-diffusion (or BAJD) as a special case, in which the Lévy process $\left(J_{t}, t \geq 0\right)$ takes the form of a compound Poisson process with exponentially distributed jumps. The BAJD was introduced by Duffie and Gârleanu [2] to describe the dynamics of default intensity. It was also used in $[5,12]$ as a short-rate model. Motivated by some applications in finance, the long-time behavior of the BAJD has been well studied. According to [12, Theorem 3.16] and [10, Proposition 3.1], the BAJD possesses a unique invariant probability measure, whose distributional properties were later investigated in [9, 11]. We remark that the results in $[10,11]$ are very general and hold for a large class of affine process with state space $\mathbf{R}_{+}$, where $\mathbf{R}_{+}$denotes the set of all non-negative real numbers. The existence and some approximations of the transition densities of the BAJD can be found in [6]. A closed formula of the transition densities of the BAJD was recently derived in [9].

In this paper we are interested in two problems concerning the JCIR defined in (1). The first one is to study the transition density estimates of the JCIR. Our first main result of this paper is a lower bound on the transition densities of the JCIR. Our idea to establish the lower bound of the transition densities is as follows. Like the BAJD, the JCIR is also an affine processes in $\mathbf{R}_{+}$. Roughly speaking, affine processes are 
Markov processes for which the logarithm of the characteristic function of the process is affine with respect to the initial state. Affine processes on the canonical state space $\mathbf{R}_{+}^{m} \times \mathbf{R}^{n}$ have been investigated in $[3,5,13,14]$. Based on the exponential-affine structure of the JCIR, we are able to compute its characteristic function explicitly. Moreover, this enables us to represent the distribution of the JCIR as the convolution of two distributions. The first distribution is known and coincides with the distribution of the CIR model. However, the second distribution is more complicated. We will give a sufficient condition such that the second distribution is singular at the point 0 . In this way we derive a lower bound estimate of the transition densities of the JCIR.

The other problem we consider in this paper is the exponential ergodicity of the JCIR. According to the main results of [10] (see also [12]), the JCIR has a unique invariant probability measure $\pi$, given that some integrability condition on the Lévy measure of $\left(J_{t}, t \geq 0\right)$ is satisfied. Under some sharper assumptions we show in this paper that the convergence of the law of the JCIR process to its invariant probability measure under the total variation norm is exponentially fast, which is called the exponential ergodicity. Our method is the same as in [9], namely we show the existence of a Forster-Lyapunov function and then apply the general framework of [16-18] to get the exponential ergodicity.

The remainder of this paper is organized as follows. In Sect. 2 we collect some key facts on the JCIR and in particular derive its characteristic function. In Sect. 3 we study the characteristic function of the JCIR and prove a lower bound of its transition densities. In Sect. 4 we show the existence of a Forster-Lyapunov function and the exponential ergodicity for the JCIR.

\section{Preliminaries}

In this section we use the exponential-affine structure of the JCIR process to derive its characteristic functions.

We recall that the JCIR process $\left(X_{t}, t \geq 0\right)$ is defined to be the solution to (1) and it depends obviously on its initial value $X_{0}$. From now on we denote by $\left(X_{t}^{x}, t \geq 0\right)$ the JCIR process started from an initial point $x \geq 0$, namely

$$
d X_{t}^{x}=a\left(\theta-X_{t}^{x}\right) d t+\sigma \sqrt{X_{t}^{x}} d W_{t}+d J_{t}, \quad X_{0}^{x}=x .
$$

Since the JCIR process is an affine process, the corresponding characteristic functions of $\left(X_{t}^{x}, t \geq 0\right)$ is of exponential-affine form:

$$
E\left[e^{u X_{t}^{x}}\right]=e^{\phi(t, u)+x \psi(t, u)}, \quad u \in \mathscr{U}:=\{u \in \mathbf{C}: \Re u \leq 0\},
$$


where $\Re u$ denotes the real part of $u$ and the functions $\phi(t, u)$ and $\psi(t, u)$ in turn are given as solutions of the generalized Riccati equations

$$
\begin{cases}\partial_{t} \phi(t, u)=F(\psi(t, u)), & \phi(0, u)=0, \\ \partial_{t} \psi(t, u)=R(\psi(t, u)), & \psi(0, u)=u \in \mathscr{U}\end{cases}
$$

with the functions $F$ and $R$ given by

$$
\begin{aligned}
& F(u)=a \theta u+\int_{(0, \infty)}\left(e^{u \xi}-1\right) v(d \xi), \\
& R(u)=\frac{\sigma^{2} u^{2}}{2}-a u .
\end{aligned}
$$

Solving the system (5) gives $\phi(t, u)$ and $\psi(t, u)$ in their explicit forms:

$$
\psi(t, u)=\frac{u e^{-a t}}{1-\frac{\sigma^{2}}{2 a} u\left(1-e^{-a t}\right)}
$$

and

$$
\phi(t, u)=-\frac{2 a \theta}{\sigma^{2}} \log \left(1-\frac{\sigma^{2}}{2 a} u\left(1-e^{-a t}\right)\right)+\int_{0}^{t} \int_{(0, \infty)}\left(e^{\xi \psi(s, u)}-1\right) v(d \xi) d s .
$$

Here the complex-valued $\operatorname{logarithmic}$ function $\log (\cdot)$ is understood to be its main branch defined on $\mathbf{C} \backslash\{0\}$. For $t \geq 0$ and $u \in \mathscr{U}$ we define

$$
\begin{aligned}
\varphi_{1}(t, u, x) & :=\left(1-\frac{\sigma^{2}}{2 a} u\left(1-e^{-a t}\right)\right)^{-\frac{2 a \theta}{\sigma^{2}}} \exp \left(\frac{x u e^{-a t}}{1-\frac{\sigma^{2}}{2 a} u\left(1-e^{-a t}\right)}\right) \\
\varphi_{2}(t, u) & :=\exp \left(\int_{0}^{t} \int_{0}^{\infty}\left(e^{\xi \psi(s, u)}-1\right) v(d \xi) d s\right)
\end{aligned}
$$

where the complex-valued power function $z^{-2 a \theta / \sigma^{2}}:=\exp \left(-\left(2 a \theta / \sigma^{2}\right) \log z\right)$ is also understood to be its main branch defined on $\mathbf{C} \backslash\{0\}$. One can notice that $\varphi_{2}(t, u)$ resembles the characteristic function of a compound Poisson distribution.

It follows from (4), (6) and (7) that the characteristic functions of $\left(X_{t}^{x}, t \geq 0\right)$ is given by

$$
E\left[e^{u X_{t}^{x}}\right]=\varphi_{1}(t, u, x) \varphi_{2}(t, u), \quad u \in \mathscr{U},
$$

where $\varphi_{1}(t, u, x)$ and $\varphi_{2}(t, u)$ are defined by (8).

According to the parameters of the JCIR process we look at two special cases: 


\subsection{Special Case (i): v = 0, No Jumps}

Notice that the case $v=0$ corresponds to the classical CIR model $\left(Y_{t}, t \geq 0\right)$ satisfying the following stochastic differential equation

$$
d Y_{t}^{x}=a\left(\theta-Y_{t}^{x}\right) d t+\sigma \sqrt{Y_{t}^{x}} d W_{t}, \quad Y_{0}^{x}=x \geq 0 .
$$

It follows from (9) that the characteristic function of $\left(Y_{t}^{x}, t \geq 0\right)$ coincides with $\varphi_{1}(t, u, x)$, namely for $u \in \mathscr{U}$

$$
E\left[e^{u Y_{t}^{x}}\right]=\varphi_{1}(t, u, x)
$$

It is well known that the classical CIR model $\left(Y_{t}^{x}, t \geq 0\right)$ has transition density functions $f(t, x, y)$ given by

$$
f(t, x, y)=\kappa e^{-u-v}\left(\frac{v}{u}\right)^{\frac{q}{2}} I_{q}\left(2(u v)^{\frac{1}{2}}\right)
$$

for $t>0, x>0$ and $y \geq 0$, where

$$
\begin{array}{ll}
\kappa \equiv \frac{2 a}{\sigma^{2}\left(1-e^{-a t}\right)}, & u \equiv \kappa x e^{-a t}, \\
v \equiv \kappa y, & q \equiv \frac{2 a \theta}{\sigma^{2}}-1,
\end{array}
$$

and $I_{q}(\cdot)$ is the modified Bessel function of the first kind of order $q$. For $x=0$ the formula of the density function $f(t, x, y)$ is given by

$$
f(t, 0, y)=\frac{c}{\Gamma(q+1)} v^{q} e^{-v}
$$

for $t>0$ and $y \geq 0$.

\subsection{Special Case (ii): $\theta=0$ and $x=0$}

We denote by $\left(Z_{t}, t \geq 0\right)$ the JCIR process given by

$$
d Z_{t}=-a Z_{t} d t+\sigma \sqrt{Z_{t}} d W_{t}+d J_{t}, \quad Z_{0}=0 .
$$

In this particular case the characteristic functions of $\left(Z_{t}, t \geq 0\right)$ is equal to $\varphi_{2}(t, u)$, namely for $u \in \mathscr{U}$

$$
E\left[e^{u Z_{t}}\right]=\varphi_{2}(t, u)
$$




\section{A Lower Bound for the Transition Densities of JCIR}

In this section we will find some conditions on the Lévy measure $v$ of $\left(J_{t}, t \geq 0\right)$ such that an explicit lower bound for the transition densities of the JCIR process given in (3) can be derived. As a first step we show that the law of $X_{t}^{x}, t>0$, in (3) is absolutely continuous with respect to the Lebesgue measure and thus possesses a density function.

Lemma 1 Consider the JCIR process $\left(X_{t}^{x}, t \geq 0\right.$ ) (started from $x \geq 0$ ) that is defined in (3). Then for any $t>0$ and $x \geq 0$ the law of $X_{t}^{x}$ is absolutely continuous with respect to the Lebesgue measure and thus possesses a density function $p(t, x, y), y \geq 0$.

Proof As shown in the previous section, it holds

$$
E\left[e^{u X_{t}^{x}}\right]=\varphi_{1}(t, u, x) \varphi_{2}(t, u)=E\left[e^{u Y_{t}^{x}}\right] E\left[e^{u Z_{t}}\right]
$$

therefore the law of $X_{t}^{x}$, denoted by $\mu_{X_{t}^{x}}$, is the convolution of the laws of $Y_{t}^{x}$ and $Z_{t}$. Since $\left(Y_{t}^{x}, t \geq 0\right)$ is the well-known CIR process and has transition density functions $f(t, x, y), t>0, x, y \geq 0$ with respect to the Lebesgue measure, thus $\mu_{X_{t}^{x}}$ is also absolutely continuous with respect to the Lebesgue measure and possesses a density function.

In order to get a lower bound for the transition densities of the JCIR process we need the following lemma.

Lemma 2 Suppose that $\int_{(0,1)} \xi \ln (1 / \xi) v(d \xi)<\infty$. Then $\varphi_{2}$ defined by (8) is the characteristic function of a compound Poisson distribution. In particular, $P\left(Z_{t}=\right.$ $0)>0$ for all $t>0$, where $\left(Z_{t}, t \geq 0\right)$ is defined by (14).

Proof It follows from (6), (8) and (15) that

$$
E\left[e^{u Z_{t}}\right]=\varphi_{2}(t, u)=\exp \left(\int_{0}^{t} \int_{(0, \infty)}\left(\exp \left(\frac{\xi u e^{-a s}}{1-\left(\sigma^{2} / 2 a\right)\left(1-e^{-a s}\right) u}\right)-1\right) v(d \xi) d s\right)
$$

where $u \in \mathscr{U}$. Define

$$
\Delta:=\int_{0}^{t} \int_{(0, \infty)}\left(\exp \left(\frac{\xi u e^{-a s}}{1-\left(\sigma^{2} / 2 a\right)\left(1-e^{-a s}\right) u}\right)-1\right) v(d \xi) d s .
$$

If we rewrite

$$
\exp \left(\frac{\xi e^{-a s} u}{1-\left(\sigma^{2} / 2 a\right)\left(1-e^{-a s}\right) u}\right)=\exp \left(\frac{\alpha u}{\beta-u}\right)
$$


where

$$
\left\{\begin{array}{l}
\alpha:=\frac{2 a \xi}{\sigma^{2}\left(e^{a s}-1\right)}>0, \\
\beta:=\frac{2 a e^{a s}}{\sigma^{2}\left(e^{a s}-1\right)}>0
\end{array}\right.
$$

then we recognize that the right-hand side of (16) is the characteristic function of a Bessel distribution with parameters $\alpha$ and $\beta$. Recall that a probability measure $\mu_{\alpha, \beta}$ on $\left(\mathbf{R}_{+}, \mathscr{B}\left(\mathbf{R}_{+}\right)\right)$is called a Bessel distribution with parameters $\alpha$ and $\beta$ if

$$
\mu_{\alpha, \beta}(d x)=e^{-\alpha} \delta_{0}(d x)+\beta e^{-\alpha-\beta x} \sqrt{\frac{\alpha}{\beta x}} I_{1}(2 \sqrt{\alpha \beta x}) d x,
$$

where $\delta_{0}$ is the Dirac measure at the origin and $I_{1}$ is the modified Bessel function of the first kind, namely

$$
I_{1}(r)=\frac{r}{2} \sum_{k=0}^{\infty} \frac{\left(\frac{1}{4} r^{2}\right)^{k}}{k !(k+1) !}, \quad r \in \mathbf{R}
$$

For more properties of Bessel distributions we refer the readers to [8, Sect. 3] (see also [4, p. 438] and [9, Sect. 3]). Let $\hat{\mu}_{\alpha, \beta}$ denote the characteristic function of the Bessel distribution $\mu_{\alpha, \beta}$ with parameters $\alpha$ and $\beta$ which are defined in (17). It follows from [9, Lemma 3.1] that

$$
\hat{\mu}_{\alpha, \beta}(u)=\exp \left(\frac{\alpha u}{\beta-u}\right)=\exp \left(\frac{\xi e^{-a s} u}{1-\left(\sigma^{2} / 2 a\right)\left(1-e^{-a s}\right) u}\right) .
$$

Therefore

$$
\begin{aligned}
\Delta & =\int_{0}^{t} \int_{(0, \infty)}\left(\hat{\mu}_{\alpha, \beta}(u)-1\right) v(d \xi) d s \\
& =\int_{0}^{t} \int_{(0, \infty)}\left(e^{\frac{\alpha u}{\beta-u}}-e^{-\alpha}+e^{-\alpha}-1\right) v(d \xi) d s .
\end{aligned}
$$

Set

$$
\begin{aligned}
\lambda & :=\int_{0}^{t} \int_{(0, \infty)}\left(1-e^{-\alpha}\right) v(d \xi) d s \\
& =\int_{0}^{t} \int_{(0, \infty)}\left(1-e^{-\frac{2 a \xi}{\sigma^{2}\left(e^{a s}-1\right)}}\right) v(d \xi) d s .
\end{aligned}
$$


If $\lambda<\infty$, then

$$
\begin{aligned}
\Delta & =\int_{0}^{t} \int_{(0, \infty)}\left(e^{\frac{\alpha u}{\beta-u}}-e^{-\alpha}\right) v(d \xi) d s-\lambda \\
& =\lambda\left(\frac{1}{\lambda} \int_{0}^{t} \int_{(0, \infty)}\left(e^{\frac{\alpha u}{\beta-u}}-e^{-\alpha}\right) v(d \xi) d s-1\right) .
\end{aligned}
$$

The fact that $\lambda<\infty$ will be shown later in this proof.

Next we show that the term $(1 / \lambda) \int_{0}^{t} \int_{(0, \infty)}(\exp (\alpha u /(\beta-u))-\exp (-\alpha)) v(d \xi) d s$ can be viewed as the characteristic function of a probability measure $\rho$. To define $\rho$, we first construct the following measures

$$
m_{\alpha, \beta}(d x):=\beta e^{-\alpha-\beta x} \sqrt{\frac{\alpha}{\beta x}} I_{1}(2 \sqrt{\alpha \beta x}) d x, \quad x \geq 0,
$$

where $I_{1}$ is the modified Bessel function of the first kind. Noticing that the measure $m_{\alpha, \beta}$ is the absolute continuous component of the measure $\mu_{\alpha, \beta}$ in (18), we easily get

$$
\hat{m}_{\alpha, \beta}(u)=\hat{\mu}_{\alpha, \beta}(u)-e^{-\alpha}=e^{\frac{\alpha u}{\beta-u}}-e^{-\alpha},
$$

where $\hat{m}_{\alpha, \beta}(u):=\int_{0}^{\infty} e^{u x} m_{\alpha, \beta}(d x)$ for $u \in \mathscr{U}$. Recall that the parameters $\alpha$ and $\beta$ defined by (17) depend on the variables $\xi$ and $s$. We can define a measure $\rho$ on $\mathbf{R}_{+}$ as follows:

$$
\rho:=\frac{1}{\lambda} \int_{0}^{t} \int_{(0, \infty)} m_{\alpha, \beta} v(d \xi) d s .
$$

By the definition of the constant $\lambda$ in (19) we get

$$
\begin{aligned}
\rho\left(\mathbf{R}_{+}\right) & =\frac{1}{\lambda} \int_{0}^{t} \int_{(0, \infty)} m_{\alpha, \beta}\left(\mathbf{R}_{+}\right) v(d \xi) d s \\
& =\frac{1}{\lambda} \int_{0}^{t} \int_{(0, \infty)}\left(1-e^{-\alpha}\right) v(d \xi) d s \\
& =1,
\end{aligned}
$$

i.e. $\rho$ is a probability measure on $\mathbf{R}_{+}$, and for $u \in \mathscr{U}$

$$
\begin{aligned}
\hat{\rho}(u) & =\int_{(0, \infty)} e^{u x} \rho(d x) \\
& =\frac{1}{\lambda} \int_{0}^{t} \int_{(0, \infty)}\left(e^{\frac{\alpha u}{\beta-u}}-e^{-\alpha}\right) v(d \xi) d s .
\end{aligned}
$$

Thus $\Delta=\lambda(\hat{\rho}(u)-1)$ and $E\left[e^{u Z_{t}}\right]=e^{\lambda(\hat{\rho}(u)-1)}$ is the characteristic function of a compound Poisson distribution. 
Now we verify that $\lambda<\infty$. Noticing that

$$
\begin{aligned}
\lambda & =\int_{0}^{t} \int_{(0, \infty)}\left(1-e^{-\alpha}\right) v(d \xi) d s \\
& =\int_{0}^{t} \int_{(0, \infty)}\left(1-e^{-\frac{2 a \xi}{\sigma^{2}\left(e^{a s}-1\right)}}\right) v(d \xi) d s \\
& =\int_{(0, \infty)} \int_{0}^{t}\left(1-e^{-\frac{2 a \xi}{\sigma^{2}\left(e^{a s}-1\right)}}\right) d s v(d \xi)
\end{aligned}
$$

we introduce the change of variables $\frac{2 a \xi}{\sigma^{2}\left(e^{a s}-1\right)}:=y$ and then get

$$
\begin{aligned}
d y & =-\frac{2 a \xi}{\sigma^{2}\left(e^{a s}-1\right)^{2}} a e^{a s} d s \\
& =-y^{2} \frac{\sigma^{2}}{2 \xi}\left(\frac{2 a \xi}{\sigma^{2} y}+1\right) d s .
\end{aligned}
$$

Therefore

$$
\begin{aligned}
\lambda & =\int_{(0, \infty)} v(d \xi) \int_{\infty}^{\frac{2 a \xi}{\sigma^{2}\left(e^{a t}-1\right)}}\left(1-e^{-y}\right) \frac{-2 \xi}{2 a \xi y+\sigma^{2} y^{2}} d y \\
& =\int_{(0, \infty)} v(d \xi) \int_{\frac{2 a \xi}{\sigma^{2}\left(e^{a t}-1\right)}}^{\infty}\left(1-e^{-y}\right) \frac{2 \xi}{2 a \xi y+\sigma^{2} y^{2}} d y \\
& =\int_{(0, \infty)} v(d \xi) \int_{\frac{\xi}{\delta}}^{\infty}\left(1-e^{-y}\right) \frac{2 \xi}{2 a \xi y+\sigma^{2} y^{2}} d y
\end{aligned}
$$

where $\delta:=\frac{\sigma^{2}\left(e^{a t}-1\right)}{2 a}$. Define

$$
M(\xi):=\int_{\frac{\xi}{\delta}}^{\infty}\left(1-e^{-y}\right) \frac{2 \xi}{2 a \xi y+\sigma^{2} y^{2}} d y .
$$

Then $M(\xi)$ is continuous on $(0, \infty)$. As $\xi \rightarrow 0$ we get

$$
\begin{aligned}
M(\xi) & =\int_{\frac{\xi}{\delta}}^{1}\left(1-e^{-y}\right) \frac{2 \xi}{2 a \xi y+\sigma^{2} y^{2}} d y+2 \xi \int_{1}^{\infty}\left(1-e^{-y}\right) \frac{d y}{2 a \xi y+\sigma^{2} y^{2}} \\
& \leq 2 \xi \int_{\frac{\xi}{\delta}}^{1} \frac{y}{2 a \xi y+\sigma^{2} y^{2}} d y+2 \xi \int_{1}^{\infty} \frac{1}{2 a \xi y+\sigma^{2} y^{2}} d y \\
& \leq 2 \xi \int_{\frac{\xi}{\delta}}^{1} \frac{1}{2 a \xi+\sigma^{2} y} d y+2 \xi \int_{1}^{\infty} \frac{1}{\sigma^{2} y^{2}} d y
\end{aligned}
$$


Since

$$
\begin{aligned}
2 \xi \int_{\frac{\xi}{\delta}}^{1} \frac{1}{2 a \xi+\sigma^{2} y} d y & =\frac{2 \xi}{\sigma^{2}}\left[\ln \left(2 a \xi+\sigma^{2} y\right)\right]_{\frac{\xi}{\delta}}^{1} \\
& =\frac{2 \xi}{\sigma^{2}} \ln \left(2 a \xi+\sigma^{2}\right)-\frac{2 \xi}{\sigma^{2}} \ln \left(2 a \xi+\frac{\sigma^{2} \xi}{\delta}\right) \\
& \leq c_{1} \xi+c_{2} \xi \ln \left(\frac{1}{\xi}\right) \leq c_{3} \xi \ln \left(\frac{1}{\xi}\right)
\end{aligned}
$$

for sufficiently small $\xi$, we conclude that

$$
M(\xi) \leq c_{4} \xi \ln \left(\frac{1}{\xi}\right), \quad \text { as } \xi \rightarrow 0
$$

If $\xi \rightarrow \infty$, then

$$
\begin{aligned}
M(\xi) & \leq \int_{\frac{\xi}{\delta}}^{\infty}\left(1-e^{-y}\right) \frac{2 \xi}{2 a \xi y+\sigma^{2} y^{2}} d y \\
& \leq \int_{\frac{\xi}{\delta}}^{\infty} \frac{2 \xi}{2 a \xi y+\sigma^{2} y^{2}} d y \leq 2 \xi \int_{\frac{\xi}{\delta}}^{\infty} \frac{1}{\sigma^{2} y^{2}} d y \\
& =\frac{2 \xi}{\sigma^{2}} \int_{\frac{\xi}{\delta}}^{\infty} d\left(-\frac{1}{y}\right)=\frac{2 \xi}{\sigma^{2}}\left[-\frac{1}{y}\right]_{\frac{\xi}{\delta}}^{\infty} \\
& =\frac{2 \xi}{\sigma^{2}} \frac{\delta}{\xi}=\frac{2 \delta}{\sigma^{2}}:=c_{5}<\infty
\end{aligned}
$$

Therefore,

$$
\lambda \leq c_{4} \int_{0}^{1} \xi \ln \left(\frac{1}{\xi}\right) v(d \xi)+c_{5} \int_{1}^{\infty} 1 v(d \xi)<\infty .
$$

With the help of the Lemma 2 we can easily prove the following proposition.

Proposition 1 Let $p(t, x, y), t>0, x, y \geq 0$ denote the transition density of the JCIR process $\left(X_{t}^{x}, t \geq 0\right)$ defined in (3). Suppose that $\int_{(0,1)} \xi \ln \left(\frac{1}{\xi}\right) v(d \xi)<\infty$. Then for all $t>0, x, y \geq 0$ we have

$$
p(t, x, y) \geq P\left(Z_{t}=0\right) f(t, x, y),
$$

where $P\left(Z_{t}=0\right)>0$ for all $t>0$ and $f(t, x, y)$ are transition densities of the CIR process (without jumps).

Proof According to Lemma 2, we have $P\left(Z_{t}=0\right)>0$. Since

$$
E\left[e^{u X_{t}^{x}}\right]=\varphi_{1}(t, u, x) \varphi_{2}(t, u)=E\left[e^{u Y_{t}^{x}}\right] E\left[e^{u Z_{t}}\right],
$$


the law of $X_{t}^{x}$, denoted by $\mu_{X_{t}^{x}}$, is the convolution of the laws of $Y_{t}^{x}$ and $Z_{t}$. Thus for all $A \in \mathscr{B}\left(\mathbf{R}_{+}\right)$

$$
\begin{aligned}
\mu_{X_{t}^{x}}(A) & =\int_{\mathbf{R}_{+}} \mu_{Y_{t}^{x}}(A-y) \mu_{Z_{t}}(d y) \\
& \geq \int_{\{0\}} \mu_{Y_{t}^{x}}(A-y) \mu_{Z_{t}}(d y) \\
& \geq \mu_{Y_{t}^{x}}(A) \mu_{Z_{t}}(\{0\}) \\
& \geq P\left(Z_{t}=0\right) \mu_{Y_{t}^{x}}(A) \\
& \geq P\left(Z_{t}=0\right) \int_{A} f(t, x, y) d y,
\end{aligned}
$$

where $f(t, x, y)$ are the transition densities of the classical CIR process given in (12). Since $A \in \mathscr{B}\left(\mathbf{R}_{+}\right)$is arbitrary, we get

$$
p(t, x, y) \geq P\left(Z_{t}=0\right) f(t, x, y)
$$

for all $t>0, x, y \geq 0$.

\section{Exponential Ergodicity of JCIR}

In this section we find some sufficient conditions such that the JCIR process is exponentially ergodic. We have derived a lower bound for the transition densities of the JCIR process in the previous section. Next we show that the function $V(x)=x$, $x \geq 0$, is a Forster-Lyapunov function for the JCIR process.

Lemma 3 Suppose that $\int_{(1, \infty)} \xi \nu(d \xi)<\infty$. Then the function $V(x)=x, x \geq 0$, is a Forster-Lyapunov function for the JCIR process defined in (3), in the sense that for all $t>0, x \geq 0$,

$$
E\left[V\left(X_{t}^{x}\right)\right] \leq e^{-a t} V(x)+M
$$

where $0<M<\infty$ is a constant.

Proof We know that $\mu_{X_{t}^{x}}=\mu_{Y_{t}^{x}} * \mu_{Z_{t}}$, therefore

$$
E\left[X_{t}^{x}\right]=E\left[Y_{t}^{x}\right]+E\left[Z_{t}\right]
$$

Since $\left(Y_{t}^{x}, t \geq 0\right)$ is the CIR process starting from $x$, it is known that $\mu_{Y_{t}^{x}}$ is a non-central Chi-squared distribution and thus $E\left[Y_{t}^{x}\right]<\infty$. Next we show that $E\left[Z_{t}\right]<\infty$.

Let $u \in(-\infty, 0)$. By using Fatou's Lemma we get 


$$
\begin{aligned}
E\left[Z_{t}\right] & =E\left[\lim _{u \rightarrow 0} \frac{e^{u Z_{t}}-1}{u}\right] \\
& \leq \liminf _{u \rightarrow 0} E\left[\frac{e^{u Z_{t}}-1}{u}\right]=\liminf _{u \rightarrow 0} \frac{E\left[e^{u Z_{t}}\right]-1}{u} .
\end{aligned}
$$

Recall that

$$
E\left[e^{u Z_{t}}\right]=\varphi_{2}(t, u)=\exp \left(\int_{0}^{t} \int_{(0, \infty)}\left(e^{\frac{\xi u e^{-a s}}{1-\left(\sigma^{2} / 2 a\right)\left(1-e^{-a s}\right) u}}-1\right) \nu(d \xi) d s\right)=e^{\Delta(u)} .
$$

Then we have for all $u \leq 0$

$$
\begin{aligned}
& \frac{\partial}{\partial u}\left(\exp \left(\frac{\xi u e^{-a s}}{1-\left(\sigma^{2} / 2 a\right)\left(1-e^{-a s}\right) u}\right)-1\right) \\
= & \frac{\xi e^{-a s}}{\left(1-\left(\sigma^{2} / 2 a\right)\left(1-e^{-a s}\right) u\right)^{2}} \exp \left(\frac{\xi u e^{-a s}}{1-\left(\sigma^{2} / 2 a\right)\left(1-e^{-a s}\right) u}\right) \\
\leq & \frac{\xi e^{-a s}}{\left(1-\left(\sigma^{2} / 2 a\right)\left(1-e^{-a s}\right) u\right)^{2}} \leq \xi e^{-a s}
\end{aligned}
$$

and further

$$
\int_{0}^{t} \int_{(0, \infty)} \xi e^{-a s} v(d \xi) d s<\infty
$$

Thus $\Delta(u)$ is differentiable in $u$ and

$$
\Delta^{\prime}(0)=\int_{0}^{t} \int_{(0, \infty)} \xi e^{-a s} v(d \xi) d s=\frac{1-e^{-a t}}{a} \int_{(0, \infty)} \xi v(d \xi) .
$$

It follows that

$$
\begin{aligned}
E\left[Z_{t}\right] & \leq \liminf _{u \rightarrow 0} \frac{\varphi_{2}(t, u)-\varphi_{2}(t, 0)}{u} \\
& =\left.\frac{\partial \varphi_{2}(t, u)}{\partial u}\right|_{u=0}=e^{\Delta(0)} \Delta^{\prime}(0) \\
& =\frac{1-e^{-a t}}{a} \int_{(0, \infty)} \xi v(d \xi) .
\end{aligned}
$$

Therefore under the assumption $\int_{(0, \infty)} \xi \nu(d \xi)<\infty$ we have proved that $E\left[Z_{t}\right]<$ $\infty$. Furthermore,

$$
E\left[Z_{t}\right]=\left.\frac{\partial}{\partial u}\left(E\left[e^{u Z_{t}}\right]\right)\right|_{u=0}=\frac{1-e^{-a t}}{a} \int_{(0, \infty)} \xi \nu(d \xi)
$$


On the other hand,

$$
E\left[e^{u Y_{t}^{x}}\right]=\left(1-\left(\sigma^{2} / 2 a\right) u\left(1-e^{-a t}\right)\right)^{-2 a \theta / \sigma^{2}} \exp \left(\frac{x u e^{-a t}}{1-\left(\sigma^{2} / 2 a\right) u\left(1-e^{-a t}\right)}\right)
$$

With a similar argument as above we get

$$
E\left[Y_{t}^{x}\right]=\left.\frac{\partial}{\partial u}\left(E\left[e^{u Y_{t}^{x}}\right]\right)\right|_{u=0}=\theta\left(1-e^{-a t}\right)+x e^{-a t}
$$

Altogether we get

$$
\begin{aligned}
E\left[X_{t}^{x}\right] & =E\left[Y_{t}^{x}\right]+E\left[Z_{t}\right] \\
& =\left(1-e^{-a t}\right)\left(\theta+\frac{1-e^{-a t}}{a}\right)+x e^{-a t} \\
& \leq \theta+\frac{1}{a}+x e^{-a t},
\end{aligned}
$$

namely

$$
E\left[V\left(X_{t}^{x}\right)\right] \leq \theta+\frac{1}{a}+e^{-a t} V(x)
$$

Remark 1 If $\int_{(1, \infty)} \xi v(d \xi)<\infty$, then there exists a unique invariant probability measure for the JCIR process. This fact follows from [12, Theorem 3.16] and [10, Proposition 3.1].

Let $\|\cdot\|_{T V}$ denote the total-variation norm for signed measures on $\mathbf{R}_{+}$, namely

$$
\|\mu\|_{T V}=\sup _{A \in \mathscr{B}\left(\mathbf{R}_{+}\right)}\{|\mu(A)|\} .
$$

Let $P^{t}(x, \cdot):=P\left(X_{t}^{x} \in \cdot\right)$ be the distribution of the JCIR process at time $t$ started from the initial point $x \geq 0$. Now we prove the main result of this paper.

Theorem 1 Assume that

$$
\int_{(1, \infty)} \xi v(d \xi)<\infty \text { and } \int_{(0,1)} \xi \ln \left(\frac{1}{\xi}\right) v(d \xi)<\infty .
$$

Let $\pi$ be the unique invariant probability measure for the JCIR process. Then the JCIR process is exponentially ergodic, namely there exist constants $0<\beta<1$ and $0<B<\infty$ such that

$$
\left\|P^{t}(x, \cdot)-\pi\right\|_{T V} \leq B(x+1) \beta^{t}, \quad t \geq 0, \quad x \in \mathbf{R}_{+} .
$$

Proof Basically, we follow the proof of [18, Theorem 6.1]. For any $\delta>0$ we consider the $\delta$-skeleton chain $\eta_{n}^{x}:=X_{n \delta}^{x}, n \in \mathbf{Z}_{+}$, where $\mathbf{Z}_{+}$denotes the set of all 
non-negative integers. Then $\left(\eta_{n}^{x}\right)_{n \in \mathbf{Z}_{+}}$is a Markov chain on the state space $\mathbf{R}_{+}$with transition kernel $P^{\delta}(x, \cdot)$ and starting point $\eta_{0}^{x}=x$. It is easy to see that the measure $\pi$ is also an invariant probability measure for the chain $\left(\eta_{n}^{x}\right)_{n \in \mathbf{Z}_{+}}, x \geq 0$.

Let $V(x)=x, x \geq 0$. It follows from the Markov property and Lemma 3 that

$$
E\left[V\left(\eta_{n+1}^{x}\right) \mid \eta_{0}^{x}, \eta_{1}^{x}, \ldots, \eta_{n}^{x}\right]=\int_{\mathbf{R}_{+}} V(y) P^{\delta}\left(\eta_{n}^{x}, d y\right) \leq e^{-a \delta} V\left(\eta_{n}^{x}\right)+M,
$$

where $M$ is a positive constant. If we set $V_{0}:=V$ and $V_{n}:=V\left(\eta_{n}^{x}\right), n \in \mathbf{N}$, then

$$
E\left[V_{1}\right] \leq e^{-a \delta} V_{0}(x)+M
$$

and

$$
E\left[V_{n+1} \mid \eta_{0}^{x}, \eta_{1}^{x}, \ldots, \eta_{n}^{x}\right] \leq e^{-a \delta} V_{n}+M, \quad n \in \mathbf{N}
$$

Now we proceed to show that the chain $\left(\eta_{n}^{x}\right)_{n \in \mathbf{Z}_{+}}, x \geq 0$, is $\lambda$-irreducible, strong aperiodic, and all compact subsets of $\mathbf{R}_{+}$are petite for the chain $\left(\eta_{n}^{x}\right)_{n \in \mathbf{Z}_{+}}$.

" $\lambda$-irreducibility": We show that the Lebesgue measure $\lambda$ on $\mathbf{R}_{+}$is an irreducibility measure for $\left(\eta_{n}^{x}\right)_{n \in \mathbf{Z}_{+}}$. Let $A \in \mathscr{B}\left(\mathbf{R}_{+}\right)$and $\lambda(A)>0$. Then it follows from Proposition 1 that

$$
P\left[\eta_{1}^{x} \in A \mid \eta_{0}^{x}=x\right]=P\left(X_{\delta}^{x} \in A\right) \geq P\left(Z_{\delta}=0\right) \int_{A} f(\delta, x, y) d y>0,
$$

since $f(\delta, x, y)>0$ for any $x \in \mathbf{R}_{+}$and $y>0$. This shows that the chain $\left(\eta_{n}^{x}\right)_{n \in \mathbf{Z}_{+}}$ is irreducible with $\lambda$ being an irreducibility measure.

"Strong aperiodicity"(see [16, p. 561] for a definition): To show the strong aperiodicity of $\left(\eta_{n}^{x}\right)_{n \in \mathbf{Z}_{0}}$, we need to find a set $B \in \mathscr{B}\left(\mathbf{R}_{+}\right)$, a probability measure $m$ with $m(B)=1$, and $\varepsilon>0$ such that

$$
L(x, B)>0, \quad x \in \mathbf{R}_{+},
$$

and

$$
P\left(\eta_{1}^{x} \in A\right) \geq \varepsilon m(A), \quad x \in C, \quad A \in \mathscr{B}\left(\mathbf{R}_{+}\right),
$$

where $L(x, B):=P\left(\eta_{n}^{x} \in B\right.$ for some $\left.n \in \mathbf{N}\right)$. To this end set $B:=[0,1]$ and $g(y):=\inf _{x \in[0,1]} f(\delta, x, y), y>0$. Since for fixed $y>0$ the function $f(\delta, x, y)$ is strictly positive and continuous in $x \in[0,1]$, thus we have $g(y)>0$ and $0<$ $\int_{(0,1]} g(y) d y \leq 1$. Define

$$
m(A):=\frac{1}{\int_{(0,1]} g(y) d y} \int_{A \cap(0,1]} g(y) d y, \quad A \in \mathscr{B}\left(\mathbf{R}_{+}\right) .
$$


Then for any $x \in[0,1]$ and $A \in \mathscr{B}\left(\mathbf{R}_{+}\right)$we get

$$
\begin{aligned}
P\left(\eta_{1}^{x} \in A\right) & =P\left(X_{\delta}^{x} \in A\right) \\
& \geq P\left(Z_{\delta}=0\right) \int_{A} f(\delta, x, y) d y \\
& \geq P\left(Z_{\delta}=0\right) \int_{A \cap(0,1]} g(y) d y \\
& \geq P\left(Z_{\delta}=0\right) m(A) \int_{(0,1]} g(y) d y,
\end{aligned}
$$

so (22) holds with $\varepsilon:=P\left(Z_{\delta}=0\right) \int_{(0,1]} g(y) d y$.

Obviously

$L(x,[0,1]) \geq P\left(\eta_{1}^{x} \in[0,1]\right)=P\left(X_{\delta}^{x} \in[0,1]\right) \geq P\left(Z_{\delta}=0\right) \int_{[0,1]} f(\delta, x, y) d y>0$

for all $x \in \mathbf{R}_{+}$, which verifies (21).

"Compact subsets are petite": We have shown that $\lambda$ is an irreducibility measure for $\left(\eta_{n}^{x}\right)_{n \in \mathbf{Z}_{+}}$. According to [16, Theorem 3.4(ii)], to show that all compact sets are petite, it suffices to prove the Feller property of $\left(\eta_{n}^{x}\right)_{n \in \mathbf{Z}_{+}}, x \geq 0$. But this follows from the fact that $\left(\eta_{n}^{x}\right)_{n \in \mathbf{Z}_{+}}$is a skeleton chain of the JCIR process, which is an affine process and possess the Feller property.

According to [16, Theorem 6.3] (see also the proof of [16, Theorem 6.1]), the probability measure $\pi$ is the only invariant probability measure of the chain $\left(\eta_{n}^{x}\right)_{n \in \mathbf{Z}_{+}}$, $x \geq 0$, and there exist constants $\beta \in(0,1)$ and $C \in(0, \infty)$ such that

$$
\left\|P^{\delta n}(x, \cdot)-\pi\right\|_{T V} \leq C(x+1) \beta^{n}, \quad n \in \mathbf{Z}_{+}, \quad x \in \mathbf{R}_{+} .
$$

Then for the rest of the proof we can proceed as in [18, p. 536] and get the inequality (20).

Acknowledgments We gratefully acknowledge the suggestions of the editors and the anonymous referee. Their valuable comments have led to a significant improvement of this manuscript. The research was supported by the Research Program "DAAD-Transformation: Kurzmaßnahmen 2012/13 Program". This research was also carried out with the support of CAS-Centre for Advanced Study, at the Norwegian Academy of Science and Letter, research program SEFE.

Open Access This chapter is distributed under the terms of the Creative Commons Attribution Noncommercial License, which permits any noncommercial use, distribution, and reproduction in any medium, provided the original author(s) and source are credited. 


\section{References}

1. Cox, J.C., Ingersoll Jr, J.E., Ross, S.A.: A theory of the term structure of interest rates. Econometrica 53(2), 385-407 (1985)

2. Duffie, D., Gârleanu, N.: Risk and valuation of collateralized debt obligations. Financ. Anal. J. 57(1), 41-59 (2001)

3. Duffie, D., Filipović, D., Schachermayer, W.: Affine processes and applications in finance. Ann. Appl. Probab. 13(3), 984-1053 (2003)

4. Feller, W.: An Introduction to Probability Theory and Its Applications, vol. II, 2nd edn. Wiley, New York (1971)

5. Filipović, D.: A general characterization of one factor affine term structure models. Finance Stoch. 5(3), 389-412 (2001)

6. Filipović, D., Mayerhofer, E., Schneider, P.: Density approximations for multivariate affine jump-diffusion processes. J. Econometrics 176(2), 93-111 (2013)

7. Fu, Z., Li, Z.: Stochastic equations of non-negative processes with jumps. Stochast. Process. Appl. 120(3), 306-330 (2010)

8. Grigelionis, B.: Thorin classes of Lévy processes and their transforms. Lith. Math. J. 48(3), 294-315 (2008)

9. Jin, P., Rüdiger, B., Trabelsi, C.: Positive Harris recurrence and exponential ergodicity of the basic affine jump-diffusion. ArXiv e-prints. http://arxiv.org/abs/1501.03638 (2015). Accessed 5 May 2015

10. Keller-Ressel, M.: Moment explosions and long-term behavior of affine stochastic volatility models. Math. Finance 21(1), 73-98 (2011)

11. Keller-Ressel, M., Mijatović, A.: On the limit distributions of continuous-state branching processes with immigration. Stochast. Process. Appl. 122(6), 2329-2345 (2012)

12. Keller-Ressel, M., Steiner, T.: Yield curve shapes and the asymptotic short rate distribution in affine one-factor models. Finance Stoch. 12(2), 149-172 (2008)

13. Keller-Ressel, M., Schachermayer, W., Teichmann, J.: Affine processes are regular. Probab. Theor. Relat. Fields 151(3-4), 591-611 (2011)

14. Keller-Ressel, M., Steiner, T.: Yield curve shapes and the asymptotic short rate distribution in affine one-factor models. Finance Stoch. 12(2), 149-172 (2008)

15. Li, Z., Ma, C.: Asymptotic properties of estimators in a stable Cox-Ingersoll-Ross model. Stochast. Process. Appl. 125(8), 3196-3233 (2015)

16. Meyn, S.P., Tweedie, R.L.: Stability of Markovian processes. I. Criteria for discrete-time chains. Adv. Appl. Probab. 24(3), 542-574 (1992)

17. Meyn, S.P., Tweedie, R.L.: Stability of Markovian processes. II. Continuous-time processes and sampled chains. Adv. Appl. Probab. 25(3), 487-517 (1993)

18. Meyn, S.P., Tweedie, R.L.: Stability of Markovian processes. III. Foster-Lyapunov criteria for continuous-time processes. Adv. Appl. Probab. 25(3), 518-548 (1993) 